\title{
Three-quarter views are subjectively good because object orientation is uncertain
}

\author{
RYOSUKE NIIMI \\ University of Tokyo, Tokyo, Japan \\ and Japan Society for the Promotion of Science, Tokyo, Japan \\ AND \\ KAZUHIKO YOKOSAWA \\ University of Tokyo, Tokyo, Japan
}

\begin{abstract}
Because the objects that surround us are three-dimensional, their appearance and our visual perception of them change depending on an object's orientation relative to a viewpoint. One of the most remarkable effects of object orientation is that viewers prefer three-quarter views over others, such as front and back, but the exact source of this preference has not been firmly established. We show that object orientation perception of the threequarter view is relatively imprecise and that this impreciseness is related to preference for this view. Human vision is largely insensitive to variations among different three-quarter views (e.g., $45^{\circ}$ vs. $50^{\circ}$ ); therefore, the three-quarter view is perceived as if it corresponds to a wide range of orientations. In other words, it functions as the typical representation of the object.
\end{abstract}

If you wanted to take a good picture of an object, which orientation (i.e., viewpoint) would you choose? A typical answer is the three-quarter view, the view in which an object's orientation is oblique between front and side. Pictures of products in advertisements and catalogues also tend to favor the three-quarter view. In fact, a high subjective "goodness" for a three-quarter view has been empirically documented; when people are asked to take the best pictures of objects, they choose the three-quarter view for most objects (Blanz, Tarr, \& Bülthoff, 1999; Palmer, Rosch, \& Chase, 1981; see also Verfaillie \& Boutsen, 1995). These studies have also suggested that each familiar object has a privileged orientation, referred to as the canonical view, which yields substantial advantages for a variety of cognitive tasks, including object recognition. The three-quarter views were frequently identified as canonical views.

Why is the three-quarter view so preferred? Properties associated with visual pattern goodness, such as symmetry and regularity (Grammer \& Thornhill, 1994; Møller, 1992; Sebrechts \& Garner, 1981), are not responsible, because the three-quarter view is less symmetrical than a frontal view. It has been argued that the potential reward that the object may provide, signaled by brain regions such as the amygdala, may account for affective preferences for object images (Bar \& Neta, 2007; Senior, 2003). However, this cannot explain the preference for a threequarter view over other views of a single object. To our knowledge, there is no evidence that the three-quarter view is preferred because it induces pleasant experiences and positive affect, as compared with other views.

Rather, it is likely that the preference for the threequarter view derives from its perceived typicality; namely, it functions as the most representative view of a given object for all possible orientations. Two explanations have been proposed: the critical information and the familiarity/ frequency accounts (Palmer, 1999). According to the critical information hypothesis, the three-quarter view conveys more visual information that is critical for object perception and identification. This account receives support from findings that viewers are most effective in object recognition tasks (e.g., a short latency for object naming) when the objects are presented in oblique orientations (Humphrey \& Jolicœur, 1993; Lawson \& Humphreys, 1999; Lawson, Humphreys, \& Jolicœur, 2000; Mitsumatsu \& Yokosawa, 2002). In such tasks, object recognition is poorer when objects are aligned to accidental views, such as front and top, than with oblique presentations, because object features critical to identification are obscured (e.g., doors and rear wheels are occluded in a frontal view of a car; Biederman, 1987). Also, these views may distort depth perception (e.g., foreshortening in a frontal view) and result in imprecise percepts of 3-D objects (Humphrey \& Jolicœur, 1993; Marr, 1982; Newell \& Findlay, 1997). But the critical information account does not explain why a side view (profile) is not a canonical view. In many cases, side views provide substantial information critical for efficient recognition. The second, familiarity/frequency

K.Yokosawa, yokosawa@1.u-tokyo.ac.jp 
account proposes that the three-quarter view is favored because it is the orientation that is most frequently encountered in daily life. Furthermore, repeated exposure to a stimulus has been linked to the induction of positive affect (Bornstein, 1989; Zajonc, 1968). Also, a higher presentation frequency of an object in a given orientation may contribute to more effective recognition of this object (Tarr, 1995). However, a number of exceptions cast doubt on this explanation. Shoes and personal computers are most frequently observed from top and front views, respectively. Nevertheless, even these objects look "best" in three-quarter views, as they appear in catalogs.

What else, besides critical information and familiarity/ frequency, might distinguish the three-quarter view? Recently we found that the human ability to perceive the precise orientation of objects is actually poorer for threequarter views than for front, side, and back views (Niimi $\&$ Yokosawa, 2008). Oblique orientations $\left(45^{\circ}, 135^{\circ}\right)$ are imprecisely perceived and poorly differentiated from other nearby oblique orientations. This uncertainty in object orientation perception may account for the perceived typicality of three-quarter views. If a three-quarter view largely fails to provide viewers with specific information about an object's orientation, then paradoxically, it may seem more acceptable to them, because such a misperception means that a given three-quarter view can represent a number of related orientations. In this respect, lower sensitivity to differences among oblique object orientations may render the three-quarter view the more typical view.

In this study, we tested the hypothesis that lower sensitivity to object orientation leads to judgments that reflect view-goodness ratings.

First, we conducted an orientation evaluation experiment in order to estimate the sensitivity to object orientation.
Second, different participants served in a view-goodness rating experiment designed to measure subjective goodness of object images as a function of orientation.

\section{METHOD}

\section{Stimuli}

We prepared computer-generated color images of objects. They were created using the 3-D model data of 18 common objects used in a previous study (Niimi \& Yokosawa, 2008). All the objects had salient front-back and top-bottom axes. Objects that had an elongated or thin shape (e.g., a stapler) were not included because, for such objects, the size of the images changed radically, depending on the orientation of the object. Stimulus images were rendered by Shade 7.5 software (e frontier, Inc., Tokyo). We simulated the 3-D spatial relationship between the CRT screen and the participants' viewpoint and presented the objects as if they were located just behind the screen. In other words, the images were projected onto the plane that matched the position of the screen. Object orientations were denoted as rotation angles from the front- that is, azimuth angles. We adopted angles of $9^{\circ}, 27^{\circ}, 45^{\circ}, 63^{\circ}, 81^{\circ}, 99^{\circ}, 117^{\circ}, 135^{\circ}, 153^{\circ}$, and $171^{\circ}\left(0^{\circ}=\right.$ front), for both rightward and leftward rotations. However, since there was virtually no difference between the data pertaining to rightward and leftward rotations, we averaged these two sets of data in all the analyses. Object names were also presented at the top-left corner of the screen in order to minimize any difficulty in recognition.

\section{Participants}

Ten individuals (20-33 years old) participated in the orientation evaluation experiment, and another set of 10 individuals (21-26 years old) participated in the view-goodness rating experiment. They all reported normal or corrected-to-normal vision.

\section{Procedure}

The participants observed the stimulus images binocularly, with their heads fixed by means of a chinrest (omitted in Figure 1A) in order to maintain the viewing distance at $80 \mathrm{~cm}$. In the orientation evaluation experiment, the participants were asked to observe object images and to evaluate the objects' orientations, using an adjust-
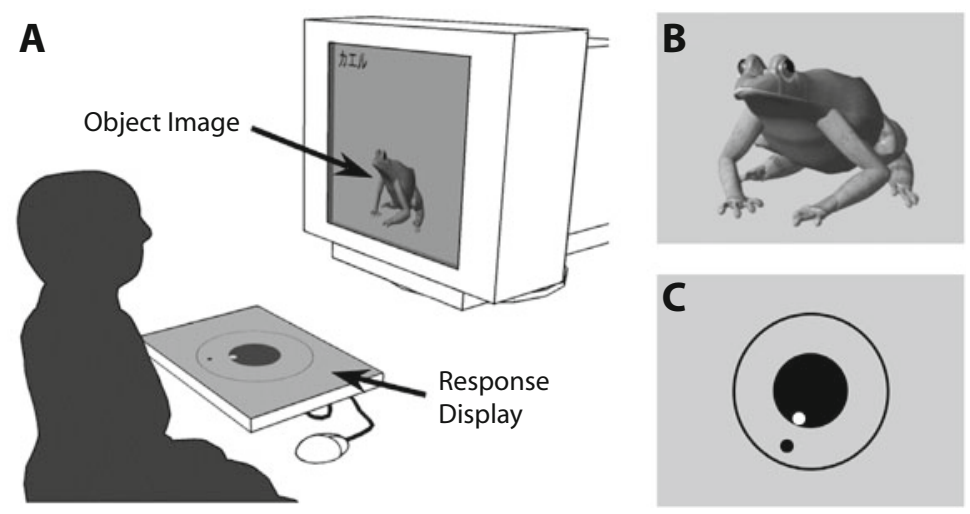

Figure 1. A setup for the orientation evaluation experiment. Human participants observed images of objects in various orientations presented on the CRT screen. (A) Participants evaluated an object's orientation using the response display disk, which was situated horizontally. (B) An example of the object image stimuli (frog in $27^{\circ}$ orientation, grayscaled in this figure). (C) The response display. Using a computer mouse, the participants rotated the dark disk to match its orientation with the object. A white dot marked the front of the disk. A mouse pointer was displayed as a black dot. When the participants clicked the mouse, the disk rotated so that the white dot was directed to the pointer (as shown in this figure). In the view-goodness rating experiment, the response display was replaced with response buttons. 

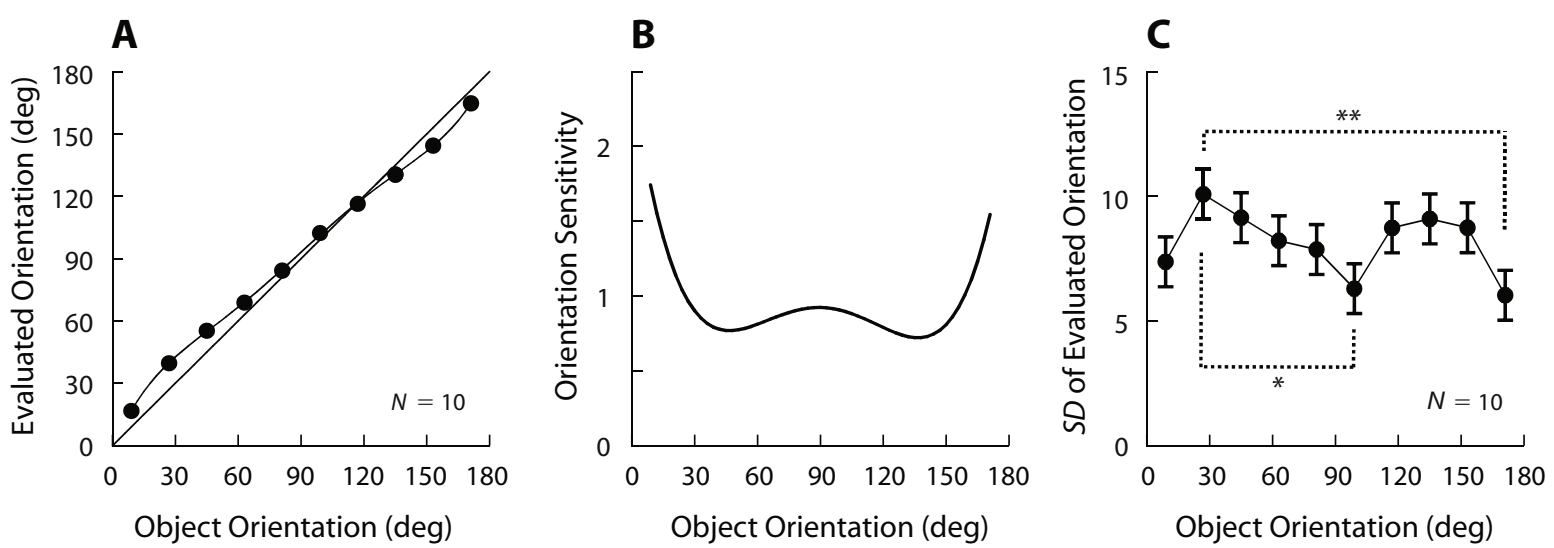

Figure 2. Results of the orientation evaluation experiment. (A) Mean evaluated orientations as a function of true object orientation (defined as rotation angles from the front). Shown is a quintic curve, fitted to the data. Perceived orientations significantly deviated from true orientations, except in the $117^{\circ}$ condition. (B) An orientation sensitivity function for all stimulus objects. The sensitivity to object orientation was estimated as the derivative of the evaluated orientation function (panel A). Oblique orientations (i.e., three-quarter views) yielded low sensitivities. (C) Standard deviations of the evaluated orientations, averaged over participants. Evaluations of oblique orientations resulted in larger $S D$ s, which indicated relatively unreliable evaluations. Error bars indicate \pm 1 standard error. $\quad * p<.05$. ${ }^{* * *} p<.01$ (multiple comparison by Tukey's HSD).

able response display disk (Figures $1 \mathrm{~A}$ and $1 \mathrm{C}$ ). They rotated the disk, using a computer mouse, and matched its orientation to the object orientations. We adopted the disk display in order to exclude salient straight edges from the response display. Stimulus images were presented in random order. The participants were instructed to respond as accurately as possible. In the view-goodness rating experiments, the response display was replaced with a keypad. The participants observed the same set of object images and answered the question "how well does the picture depict the object?" by pressing one of seven keys (e.g., $1=$ very bad, $7=$ very good $)$. No time limits for responding were imposed in any experiment.

\section{RESULTS}

For the orientation evaluation experiment, responses that showed extreme deviations ( $45^{\circ}$ or larger) from true orientations were treated as errors and were excluded from the following analysis (1.4\% of all the trials). Mean estimated settings of orientations differed significantly from the actual orientations (Figure 2A). Two-tailed $t$ tests at each of the 10 orientation points showed that mean evaluated orientations differed significantly $(p<.05)$ from true orientations, except for the orientation of $117^{\circ}$. A strong bias toward a side view $\left(90^{\circ}\right)$ was found for oblique orientations (e.g., $27^{\circ}$ was evaluated as $39.7^{\circ}$ ), indicating that orientation differences from the front $\left(0^{\circ}\right)$ and back $\left(180^{\circ}\right)$ were overestimated, because front and back views are accidental views and three-quarter views are very dissimilar from them. We replicated this bias in another experiment in which the response disk was replaced by an arrow (data not shown), thus indicating that the bias was not due to response mode.

Sensitivity to object orientation can be estimated as a derivative of the evaluated orientation function (e.g., a shallow slope indicates that the magnitude of change in object orientation is underestimated). The resulting derivative, the orientation sensitivity function (Figure 2B), shows that three- quarter views $\left(45^{\circ}, 135^{\circ}\right.$, and nearby oblique orientations) involve lower orientation sensitivities. Note that the sensitivity value does not reflect the absolute deviation of the perceived orientation from the true orientation but, instead, reflects the participants' ability to detect small orientation changes. The pattern of the sensitivity function indicates that the participants tended to underestimate the orientation differences among the oblique orientations (as reflected by sensitivity values below 1). For example, the estimated sensitivity for the $45^{\circ}$ orientation was 0.77 , which means that the participants perceived only a $0.77^{\circ}$ change in object orientation when the stimulus objects were rotated $1^{\circ}$ from a $45^{\circ}$ orientation. This pattern of results is consistent with previous findings (Niimi \& Yokosawa, 2008). We observed larger standard deviations for the evaluation of oblique orientations (Figure 2C), which also suggests an imprecise and unreliable orientation perception for the three-quarter view. In contrast, orientations near the front and back yielded high sensitivities (larger than 1), suggesting that the participants overestimated the orientation difference from the front and back. The side orientation $\left(90^{\circ}\right)$ provided a sensitivity value close to 1 . This means that the participants correctly evaluated the orientation changes from the side (neither underestimation nor overestimation). We applied the above procedure of estimation of orientation sensitivity function to the data for each object and estimated orientation sensitivity values for the 180 instances ( 18 objects $X$ 10 orientations).

Mean rated score from the view-goodness rating experiment (Figure $3 \mathrm{~A}$ ) showed that three-quarter views, such as $27^{\circ}$ and $45^{\circ}$, were rated better than other views. In addition, a strong tendency to rate near-front orientations better than near-back orientations was found, probably because frontal views are more frequently encountered in daily life or contain subjectively more important parts, such as facial features, as compared with back views. Thus, 
A

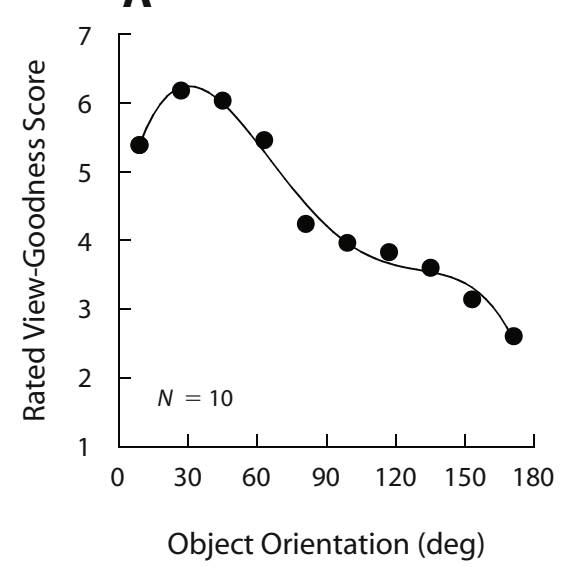

Figure 3. Result of the view-goodness rating experiment. (A) A view-goodness function for all objects. Shown is a quartic curve, fitted to the data. This result confirmed that a three-quarter view between front and side produces maximum view-goodness. In general, rated view-goodness was lower as objects rotated toward $180^{\circ}$ (back). (B) We interpreted the function as the sum of two effects: the advantage of oblique orientations (upper panel) and the general advantage of near-front orientations over near-back orientations (lower panel). The former may be accounted for by lower orientation sensitivity for oblique orientations (Figure 2B).

the "best" views are determined by two factors: low orientation sensitivity in oblique orientations and the general advantage of frontal orientation over back orientation. This explains the skewed M-shape of the view-goodness function (see Figure 3B).

\section{Analysis}

The conclusion above was confirmed by a multiple regression analysis on the view-goodness ratings of the 180 instances (18 objects $\times 10$ orientations). Two independent variables, orientation sensitivity (estimated for each instance; see the Results section) and orientation ( $9^{\circ}-$ $171^{\circ}$ ), significantly accounted for variance in the viewgoodness ratings $\left(R^{2}=.56, p<.01, N=180\right)$. Lower orientation sensitivity was significantly related to higher goodness ratings $(B=-0.50, \beta=-0.14, p<.01)$, whereas smaller rotation angles, from the front, reliably covaried with higher goodness ratings $(B=-0.02, \beta=$ $-0.75, p<.01$ ). Virtually identical results were obtained for the data on the basis of the orientation evaluation experiment in which the response display involved an arrow $\left(R^{2}=.55, p<.01, N=180\right)$. In short, the three-quarter view is best because object orientation is oblique and close to the front.

We also investigated the direct relationship between the orientations in which orientation sensitivity was minimal and the orientations of the "best" views. First, for each object, we determined the object's orientation that was associated with the maximum of its view-goodness function. Second, we estimated a minimum-sensitivity orientation for each object; this was taken to be the orientation for which the orientation sensitivity function for that object had the lowest sensitivity value. For a majority of the objects (14 out of 18), we observed W-shaped sensitivity functions (similar to that in Figure 2B), where we identified two minimum-sensitivity orientations for each object. They corresponded closely to the front-side three-quarter view and the back-side three-quarter view (on average, $44.9^{\circ}$ and $139.6^{\circ}$, respectively). The former minimum-sensitivity orientation (i.e., the one closer to the front) may predict the canonical views. The remaining 4 objects had orientation sensitivity functions that had a single minimum, so we simply determined their minimumsensitivity orientations. These 4 objects had minimumsensitivity orientations relatively biased toward the back, and interestingly, their maximum-goodness orientations also were biased toward the back, as compared with the other 14 objects (Figure 4).

The results showed that by using minimum-sensitivity orientations, we could reliably predict the "best" orientations. The minimum-sensitivity orientations (we selected the one closer to the front for the 14 objects that had two minima) were significantly correlated with the maximum-goodness orientations $(r=.76, p<.01, N=$ 18; Figure 4). The replication experiment using an arrow display yielded a comparable result $(r=.60, p<.01, N=$ 18; data not shown). These correlations further support the conclusion that orientation sensitivity accounts for view-goodness. We also found that there are two classes of objects. For most objects, orientation sensitivity is minimal at the two three-quarter views, and the frontside three-quarter views correspond to the "best" views because near-front orientations are subjectively better than

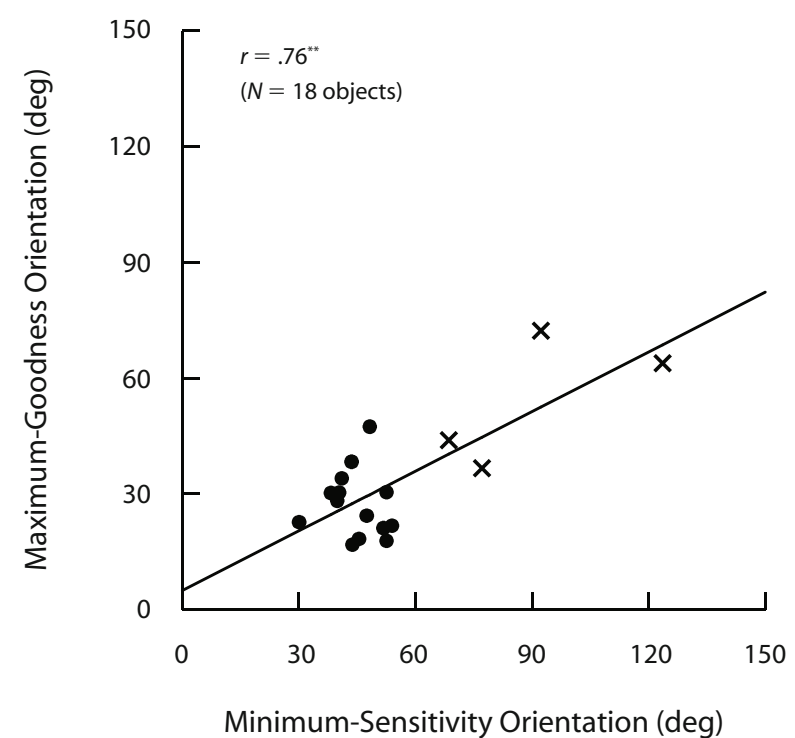

Figure 4. Correlations between maximum-goodness orientations and minimum-sensitivity orientations estimated for each object. For objects with two minimum-sensitivity orientations (plotted with dots), we used the orientation closer to the front. The remaining four objects yielded single minimum-sensitivity orientations (plotted with $\times$ ). Minima in the orientation sensitivity functions predicted the subjectively "best" views. ${ }^{* *} p<.01$. 
near-back orientations in general (discussed above). For the exceptional objects, the "best" views may be predicted as the orientations slightly rotated toward the front from the point of the objects' minimum orientation sensitivity (as reflected in the shallow slope of the line shown in Figure 4). Thus, the "best" orientations correlate with but are not identical to the minimum-sensitivity orientations. This divergence is due to the general advantage of the front.

\section{DISCUSSION}

Our results demonstrate that the higher rated viewgoodness of the three-quarter view is accounted for by the corresponding lower sensitivity to object orientation. Since this finding is based on correlation data, we should be cautious in speculating about causality. Nevertheless, we suggest that this lower orientation sensitivity is the cause of the perceived typicality of the three-quarter view. It is unlikely that the participants were relatively uncertain about the object orientation in the three-quarter views simply because they are "good" views.

Why does low sensitivity to object orientation increase one's sense of goodness? Even if an object is rotatedsay, $10^{\circ}$-from the view having lowest sensitivity, few changes in its image would be perceived, because low sensitivity implies that a wide range of orientations (e.g., $20^{\circ}-70^{\circ}$ ) will provide views that are subjectively similar. In other words, an orientation that elicits low sensitivity may be perceived to be representative of a variety of other orientations. In turn, this representative aspect of oblique orientations mediates goodness judgments, leading to an increase of view-goodness scores for three-quarter views. This is not the case with accidental views (e.g., the front), which are characteristically dissimilar from other views, having a number of qualities that are orientation specific (e.g., occlusions of parts, symmetric contours). As a result, orientation sensitivity is high for accidental views, and their view-goodness ratings are relatively low. Such views would be perceived as atypical instances.

This account of the role played by the lower orientation sensitivity for the three-quarter views may also explain why amount of critical information and familiarity (see the introduction) have been considered to be determinants of view-goodness in previous studies. First, with regard to the critical information hypothesis, low orientation sensitivity implies that three-quarter views convey orientationindependent visual information. Visual features that appear in object images independently of object orientation are ideal for object recognition, which should be robust over variation in views due to changes in orientation (Biederman, 1987, 2001; Biederman \& Bar, 1999). We speculate that orientation sensitivity correlates inversely with the efficiency of object recognition (e.g., accidental views are associated with higher orientation sensitivity and inefficient recognition, whereas canonical views are associated with lower orientation sensitivity and efficient recognition). Second, with respect to the familiarity/ frequency hypothesis, if the visual system effectively ignores variation among oblique views and, thereby, perceives a single three-quarter view as representative of a wide range of object orientations, the three-quarter view will inevitably be experienced as a more frequent view and, thus, will seem quite familiar to viewers (even if they encounter objects from all orientations equally often).

In conclusion, because lower sensitivity, in a sense, reflects a greater uncertainty regarding an object's precise orientation, enhanced uncertainty may be responsible for people's subjective view-goodness of object images. If typicality is inversely related to uncertainty, as we suggest, the more uncertain a viewer is about an object's orientation, the more typical the object's image will seem.

Furthermore, this finding may explain why accidental views, rather than three-quarter views, have often been used in artistic photographs. Note that view-goodness, as assessed in our studies, may reflect the typicality of object images but may not coincide with the artistic value. For example, the photograph of M. Duchamp's work "Fountain," taken by A. Stieglitz, is a top view of a common object. That particular orientation was necessary to produce the artistic value, because it yielded a peculiar view that any other orientation might not. If the orientation was slightly changed, the view would be altered significantly, and the artistic value would disappear. Avoidance of typical and ordinary views (which embody low orientation sensitivity and are suitable for commercial catalogs) offers opportunities to discover novel, astonishing aspects of common objects. Hence, views that have high orientation sensitivity have a potential for artistic values. Whichever value is considered, typicality or artistic novelty, perceptual uncertainty may be a key to understanding the aesthetics aspect of human vision.

\section{AUTHOR NOTE}

This study was supported by Grants-in-Aid for Scientific Research from the Japan Society for the Promotion of Science (awarded to both R.N. and K.Y.). The authors thank Irina Harris and an anonymous reviewer for helpful comments on the manuscript. Correspondence concerning this article should be addressed to K. Yokosawa, Department of Psychology, Graduate School of Humanities and Sociology, University of Tokyo, 7-3-1 Hongo, Bunkyo-ku, Tokyo 113-0033, Japan (e-mail: yokosawa@1.u-tokyo.ac.jp).

\section{REFERENCES}

BAR, M., \& NeTA, M. (2007). Visual elements of subjective preference modulate amygdala activation. Neuropsychologia, 45, 2191-2200.

BiEDERMAN, I. (1987). Recognition-by-components: A theory of human image understanding. Psychological Review, 94, 115-147.

Biederman, I. (2001). Recognizing depth-rotated objects: A review of recent research and theory. Spatial Vision, 13, 241-253.

Biederman, I., \& BAR, M. (1999). One-shot viewpoint invariance in matching novel objects. Vision Research, 39, 2885-2899.

Blanz, V., Tarr, M. J., \& Bülthoff, H. H. (1999). What object attributes determine canonical views? Perception, 28, 575-599.

BoRNSTEIN, R. F. (1989). Exposure and affect: Overview and meta-analysis of research, 1968-1987. Psychological Bulletin, 106, 265-289.

Grammer, K., \& Thornhill, R. (1994). Human (Homo sapiens) facial attractiveness and sexual selection: The role of symmetry and averageness. Journal of Comparative Psychology, 108, 233-242.

Humphrey, G. K., \& Joliceur, P. (1993). An examination of the effects 
of axis foreshortening, monocular depth cues, and visual field on object identification. Quarterly Journal of Experimental Psychology, 46A, 137-159.

Lawson, R., \& Humphreys, G. W. (1999). The effects of view in depth on the identification of line drawings and silhouettes of familiar objects: Normality and pathology. Visual Cognition, 6, 165-195.

Lawson, R., Humphreys, G. W., \& Jolicceur, P. (2000). Combined effects of plane disorientation and foreshortening on picture naming: One manipulation or two? Journal of Experimental Psychology: Human Perception \& Performance, 26, 568-581.

MARR, D. (1982). Vision. San Francisco: Freeman.

Mitsumatsu, H., \& Yokosawa, K. (2002). How do the internal details of the object contribute to recognition? Perception, 31, 1289-1298.

MøLlER, A. P. (1992). Female swallow preference for symmetrical male sexual ornaments. Nature, 357, 238-240.

Newell, F. N., \& Findlay, J. M. (1997). The effect of depth rotation on object identification. Perception, 26, 1231-1257.

NiImI, R., \& Yokosawa, K. (2008). Determining the orientation of depth-rotated familiar objects. Psychonomic Bulletin \& Review, 15, 208-214.
Palmer, S. (1999). Vision science: Photons to phenomenology. Cambridge, MA: MIT Press.

Palmer, S., Rosch, E., \& Chase, P. (1981). Canonical perspective and the perception of objects. In J. [B.] Long \& A. [D.] Baddeley (Eds.), Attention and performance $I X$ (pp. 135-151). Hillsdale, NJ: Erlbaum.

Sebrechts, M. M., \& Garner, W. R. (1981). Stimulus-specific consequences of pattern goodness. Memory \& Cognition, 9, 41-49.

SENIOR, C. (2003). Beauty in the brain of the beholder. Neuron, 38, 525528.

TARR, M. J. (1995). Rotating objects to recognize them: A case study on the role of viewpoint dependency in the recognition of threedimensional objects. Psychonomic Bulletin \& Review, 2, 55-82.

Verfaillie, K., \& Boutsen, L. (1995). A corpus of 714 full-color images of depth-rotated objects. Perception \& Psychophysics, 57, 925-961.

ZAJONC, R. B. (1968). Attitudinal effects of mere exposure. Journal of Personality \& Social Psychology Monographs, 9, 1-27.

(Manuscript received June 1, 2008; revision accepted for publication October 30,2008 .) 